\title{
Galenus-von-Pergamon-Preis 2012
}

\section{Die Jury hat entschieden}

Für manche ist er der inoffizielle „Nobelpreis" für Pharmakologie: der Galenus-von-Pergamon-Preis. Am 18. Oktober wurde er erneut verliehen - an drei beeindruckende Preisträger.

Drei Preise für exzellente Grundlagenforschung in der Pharmakologie und herausragende pharmazeutische Innovationen am Donnerstagabend war es wieder so weit: Im Rahmen einer feierlichen Gala wurde der begehrte Galenus-von-Pergamon-Preis verliehen. Bei einer festlichen Gala wurden Gilenya ${ }^{\circledast}$ (Fingolimod) von Novartis Pharma in der Kategorie Primary Care und Zelboraf (Vemurafenib) von Roche Pharma in der Kategorie Specialist Care ausgezeichnet.

In der Kategorie Grundlagenforschung wurde das Team um Dr. Thomas Worzfeld aus Bad Nauheim für die Entwicklung eines neuen Ansatzes zur Therapie bei metastasierendem Brustkrebs geehrt.

Anders als im vergangenen Jahr hat sich die 14-köpfige Jury unter dem Vorsitz von Professor Erland Erdmann aus Köln bei ihrer Entscheidung sehr schwer getan, wie der Jury-Präsident sagte. „Alle diesjährigen Kandidaten sind Innovationen." Mit Mehrheit hätten sich die Juroren schließlich für die

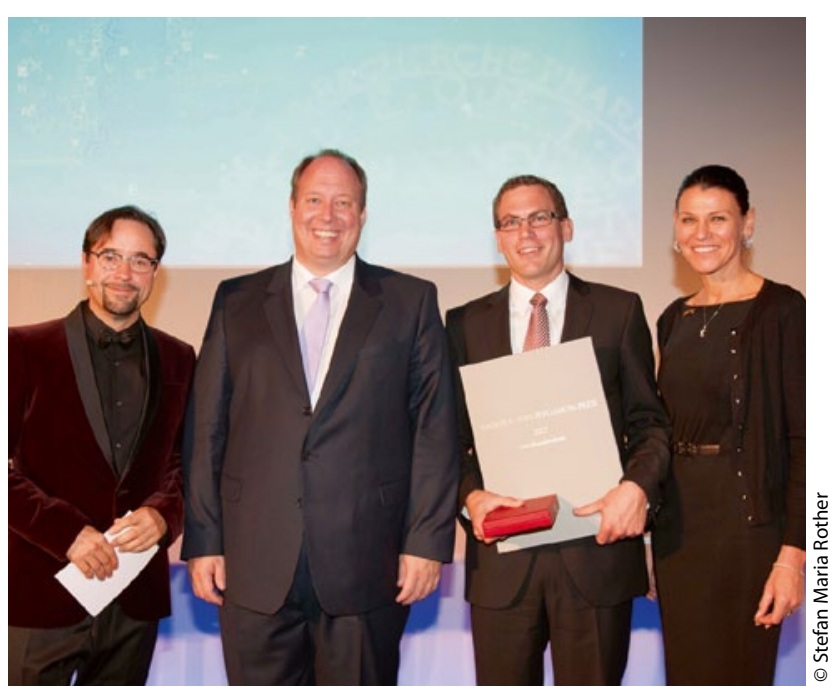

Dr. Thomas Worzfeld (2. von re.) nahm die Auszeichnung in der Kategorie Grundlagenforschung aus den Händen von Prof. Marion Kiechle, Vizepräsidentin der Galenus-Preis-Jury (re.) entgegen; mit Dr. Helge Braun (2. von li), Parlamentarischer Staatssekretär und Jan Josef Liefers (li.), Schauspieler

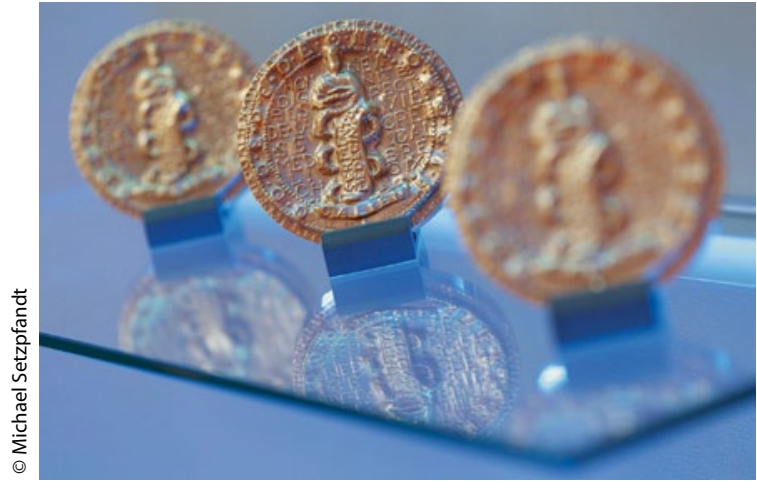

Galenus-Preisverleihung am 18. Oktober: Medaillen für die Preisträger.

diesjährigen Galenus-Gewinner entschieden. Wie wichtig Innovationen seien, betonte Harm van Maanen, Executive Vice President, Springer Medizin: „Innovationen sind eine der wichtigsten Güter, die wir haben." Deshalb sollten sie gefördert werden.

Fingolimod ist ein orales Medikament zurTherapie von Patienten mit Mulipler Sklerose (MS). Es ist zugelassen für bisher nicht behandelte Patienten, die an einer rasch fortschreitenden, schwer schubförmigen MS erkrankt sind. Außerdem ist das Präparat indiziert zur Eskalationstherapie, wenn trotz der Behandlung mit einem Beta-Interferon eine hohe Krankheitsaktivität vorliegt.

Mit Fingolimod gelingt es, die Schubrate zu verringern. Im Vergleich zur Therapie mit Interferon ließ sich die Schubrate in einer Zulassungsstudie um 52 Prozent senken $(0,33$ versus 0,16 Schübe pro Jahr).

Vemurafenib ist die erste Option für eine personalisierte Therapie bei inoperablem oder metastasiertem Melanom. Das Medikament in Form von Filmtablet- ten ist zugelassen zur Therapie von Melanom-Patienten, die ein mutiertes BRAF-Gen haben. In der Zulassungsstudie betrug die geschätzte mediane progressionsfreie Überlebenszeit 5,6 Monate im Vergleich zu Patienten mit der Standardchemotherapie mit Dacarbazin. In der Vemurafenib-Gruppe war zudem das Sterberisiko um 63 und das Progressionsrisiko um 74 Prozent verringert. Die erste Phase der Entwicklung des Präparates erfolgte durch das USUnternehmen Plexxikon.

Plexin-B1-Antikörper gegen Brustkrebs Der Galenus-Preisträger Worzfeld und sein Team haben herausgefunden, dass der Rezeptor Plexin-B1 eine besondere Bedeutung für die Metastasierung bei Brustkrebs hat. Anhand von Gewebeproben von Patientinnen mit einem Mammakarzinom stellten sie fest, dass die Frauen eine umso bessere Überlebenschance hatten, je weniger von diesem Rezeptor im Tumorgewebe vorhanden war.

Inzwischen steht ein monoklonaler Antikörper gegen diesen Rezeptor zur Verfügung, der derzeit präklinisch getestet wird. „Anfangs dachten wir nicht, dass wir mit unserer Forschung bei Brustkrebs landen würden", so Worzfeld, der die Auszeichnung aus den Händen der Galenus-Vizepräsidentin Professor Marion Kiechle aus München entgegennahm. Ausgangspunkt seien neurowissenschaftliche Fragestellungen gewesen. Geplant sind Untersuchungen mit dem Antikörper auch beim Magen- und beim Ovarialkarzinom.

Auch der Springer Medizin CharityAward 2012 ist bei der Gala verliehen worden. Mit der Auszeichnung, die Gesundheitsminister Daniel Bahr überreichte, wird die Arbeit der Einrichtung Balthasar Kinder- und Jugendhospiz in Olpe geehrt. Träger des Hospizes ist die Gemeinnützige Gesellschaft der Franziskanerinnen zu Olpe. 